\title{
Breakdown of the Yukawa model in de-ionized colloidal suspensions
}

\author{
Aldemar Torres, ${ }^{1}$ Alejandro Cuetos, ${ }^{2}$ Marjolein Dijkstra, ${ }^{2}$ and René van Roij \\ ${ }^{1}$ Institute for Theoretical Physics, Utrecht University, Leuvenlaan 4, 3584 CE Utrecht, The Netherlands \\ ${ }^{2}$ Soft Condensed Matter, Utrecht University, Princetonplein 5, 3584 CC Utrecht, The Netherlands
}

(Received 12 December 2007; published 10 March 2008)

\begin{abstract}
We study effective colloidal interactions in de-ionized colloidal mixtures through sedimentation-diffusion equilibrium. We derive a coarse-grained effective model (EM) and compare its density profiles with those of the computationally much more expensive primitive model (PM) of colloids and counterions in gravity. The EM, which contains not only standard pairwise screened-Coulomb interactions, but also explicit many-body effects by means of a so-called volume term, can quantitatively account for all observed sedimentation phenomena such as lifting of colloids to high altitudes, segregation into layers in mixtures, and floating of heavy colloids on top of lighter ones. Without the volume term there is no quantitative agreement between the PM and EM, even in the present high-temperature limit of interest, showing that de-ionized colloidal suspensions cannot be described by a pairwise Yukawa model.
\end{abstract}

DOI: 10.1103/PhysRevE.77.031402

PACS number(s): 82.70.Dd, 64.10.+h

Sedimentation of suspended colloidal particles in Earth's gravity field is often a nuisance that is to be avoided, e.g., by careful density matching the colloidal particles with the solvent or by sending samples to outer space [1]. However, it has also become clear over the last decade or so that the study of the colloidal density profile $\rho(z)$ (with $z$ the vertical height) in sedimentation-diffusion equilibrium can efficiently give quantitative information about the (osmotic) pressure $\Pi(\rho)$ of a bulk colloidal suspension at density $\rho$ and hence about the effective colloidal interactions [2-4]. In pioneering studies it was shown that this scheme, which is based on hydrostatic equilibrium, can quantitatively reproduce the known equation of state of hard spheres in the fluid phase $[2,3]$, and recent extensions show that this method also works for hard-sphere crystals and for sticky spheres [4]. In this paper we exploit the close relationship between effective colloidal interactions and sedimentation-diffusion equilibrium for the case of (mixtures of) index-matched charged colloidal spheres suspended in a dielectric solvent with monovalent salt ions, as, e.g., studied experimentally in Refs. $[5,6]$. Sedimentation of charged colloids has turned out to be interesting in its own right, given the rich phenomenology that was found for such systems during the last few yearse.g., the existence (in a conducting medium) of a macroscopic electric field that pushes the colloids up to relatively large heights [5-8] and the "colloidal Brazil nut effect" such that the heavier colloids can float on top of a layer of lighter ones [9-11]. In the regime of relatively high salt concentrations, where the ionic strength is dominated by the background electrolyte, these sedimentation phenomena have been explained by two different types of models: (i) by the multicomponent primitive model (PM) of mesoscopic charged colloids with subnanometer-sized cations and anions in a continuum solvent $[7,9]$ and (ii) by the Yukawa model (YM) of colloids interacting solely by a pairwise repulsive screened-Coulomb potential $[8,10,12]$. Advantages of the YM are that both colloid-colloid correlations and colloid-ion correlations (the "double layer") are taken into account in the hydrostatic equilibrium calculations $[8,10]$ and that larger systems can be simulated due to the absence of explicit ions.
We will show that the state of affairs is quite different at vanishingly low ionic strength: our direct simulations of the PM in gravity are not in agreement with our simulations and calculations of the YM. Given the close relationship between sedimentation profiles and the equation of state of bulk systems [2-4], this disagreement between the PM and he YM actually points to the breakdown of the YM in de-ionized bulk suspensions. We attribute this to many-body effects that become relevant at low salinity due to the long Debye length (which sets the interaction range in the YM). Instead of trying to improve the YM by extending it with triplet and higher-order potentials, such as, e.g., in Refs. [13], we focus here on the high-temperature linear screening regime and derive an explicit expression for the effective interaction Hamiltonian $W$ of the form $W=W_{1}+W_{2}$. This effective model (EM) is found to consist of the pairwise YM Hamiltonian $W_{2}$ and a so-called volume term $W_{1}$ that does not depend on colloidal coordinates, but nontrivially on the colloid densities [14]. We will show that the EM in gravity is in quantitative agreement with the PM, thereby showing that the nonpairwise many-body effects can be captured efficiently and accurately by the volume terms.

Let us consider an $n$-component suspension of colloidal species $i=1, \ldots, n$ with charges $Z_{i} e$, radii $a_{i}$, and buoyant masses $m_{i}$, confined between the bottom of a macroscopic sample cell at $z=0$ and the meniscus of the solvent at $z=H$. The colloidal chemical potentials $\mu_{i}$ are such that the average packing fraction $\bar{\eta}_{i}=\int_{0}^{H} d z \eta_{i}(z) / H$ of each species $i$ is fixed, with $\eta_{i}(z)=(4 \pi / 3) a_{i}^{3} \rho_{i}(z)$ the packing fraction of species $i$ at height $z$. The solvent is a continuum with dielectric constant $\epsilon$ at temperature $T$, such that its Bjerrum length reads $\lambda_{B}$ $=e^{2} /\left(\epsilon k_{B} T\right)$. Here $e$ is the elementary charge and $k_{B}$ the Boltzmann constant. The system is globally charge neutral due to monovalent, massless, and pointlike counterions. The gravitational potential that acts on the colloids is $m_{i} g z \equiv k_{B} T z / L_{i}$ for species $i$, with $L_{i}$ the gravitational length. The interaction potential between any pair of particles (colloids and counterions) is a sum of hard-core repulsions and (unscreened) Coulombic interactions $\propto r^{-1}$, with $r$ the center-to-center distance. 
In an earlier publication [11] we presented some direct Monte Carlo (MC) simulations of this PM in gravity. Such simulations are computationally extremely expensive and slow due to the large number of counterions per colloid and due to the long range of the Coulomb interactions. It would therefore be convenient if one could model the system as a colloids-only system by coarse-graining the ionic degrees of freedom, of course without losing quantitative agreement with the PM results. Following the local-density approximation that underlies hydrostatic equilibrium [15], the equilibrium packing fraction profiles $\eta_{i}(z)$ should satisfy

$$
k_{B} T \ln \eta_{i}(z)+\mu_{i, e x}\left(\eta_{1}(z), \ldots, \eta_{n}(z)\right)+m_{i} g z=\mu_{i},
$$

where the effective colloidal interactions enter through the excess (over ideal) chemical potentials $\mu_{i, e x}\left(\eta_{1}, \ldots, \eta_{n}\right)$ of species $i=1, \ldots, n$ in a colloidal bulk mixture. Note that Eq. (1) is equivalent, for $n=1$, to the hydrostatic equilibrium condition $d \Pi(z) / d z=-m g \rho(z)$ [15]. In order to be able to solve the $n$ local equations (1) an explicit form is needed for $\mu_{i, e x}\left(\eta_{1}, \ldots, \eta_{n}\right)$, which we obtain here by a tedious but straightforward generalization of the linear-screening theory of Ref. [16] from the one-component case $n=1$ to the multicomponent case of arbitrary $n \geq 1$.

The first step in this scheme consists of considering an arbitrary nonoverlapping fixed configuration of $N_{1}, \ldots, N_{n}$ colloids of species $i$ in a bulk volume $V$, in osmotic contact with a neutral reservoir of monovalent ions at a density $2 \rho_{s}$-at the end of the calculation we will consider $\rho_{s} \rightarrow 0$ in order to describe the de-ionized limit. The ionic density profiles $\rho_{ \pm}(\mathbf{r})=\rho_{s} \exp [\mp \phi(\mathbf{r})]$ and the electrostatic potential $k_{B} T \phi(\mathbf{r}) / e$ in the electrolyte in between the colloids follow from the Poisson-Boltzmann (PB) equation $\nabla^{2} \phi(\mathbf{r})$ $=\kappa^{2} \sinh \phi(\mathbf{r})$, where $\kappa=\left(8 \pi \lambda_{B} \rho_{s}\right)^{1 / 2}$ is the reservoir's inverse Debye screening length. By linearizing the PB equation about a self-consistent average potential $\bar{\phi}$, the PB equation can be solved and analytic expressions for $\phi(\mathbf{r})$ and $\rho_{ \pm}(\mathbf{r})$ follow as in Ref. [16]; one also finds for the so-called Donnan potential $\bar{\phi}=\operatorname{arc} \sinh \left[\rho_{c} /\left(2 \rho_{s}\right)\right]$ with the counterion density $\rho_{c}=\sum_{i=1}^{n} Z_{i} \rho_{i}$. The effective colloid Hamiltonian follows as $W=W_{1}\left(\rho_{1}, \ldots, \rho_{n}, \rho_{c}, V\right)+W_{2}\left(\rho_{1}, \ldots, \rho_{n}, \rho_{c} ;\{\mathbf{R}\}\right)$ with the so-called volume term $W_{1}$, which is independent of the colloidal coordinates, and the pairwise additive term $W_{2}$ of effective colloidal potentials of the screened-Coulomb form $v_{i j}(r)=Z_{i}^{*} Z_{j}^{*} k_{B} T \lambda_{B} \exp (-\bar{\kappa} r) / r$ between a pair of species $i$ and $j$ at separation $r>a_{i}+a_{j}$. Here $Z_{i}^{*}$ $=Z_{i} \exp \left(\bar{\kappa} a_{i}\right) /\left(1+\bar{\kappa} a_{i}\right)$ and $\bar{\kappa}=\kappa \sqrt{\cosh \bar{\phi}}$. In the limit $\rho_{s} \rightarrow 0$ one finds $\bar{\kappa}=\sqrt{4 \pi \lambda_{B} \rho_{c}}$-i.e., only the counterions contribute to the screening—and $W_{1}=V w_{1}$ with

$$
\frac{w_{1}}{k_{B} T}=\rho_{c}\left(\ln \frac{\rho_{c}}{\rho_{s}}-1\right)-\frac{1}{2} \sum_{i=1}^{n} \rho_{i}\left(\frac{Z_{i}^{2} \bar{\kappa} \lambda_{B}}{1+\bar{\kappa} a_{i}}+Z_{i}\right),
$$

where we recognize the ideal-gas entropy of the counterions and the self-energy of the colloids. Note that Eq. (2) reduces, for $n=1$, to the results of Ref. [16]. With the explicit analytic form of the effective Hamiltonian $W=W_{1}+W_{2}$ at hand we can take the next step in the calculation of $\mu_{i, e x}$. We calculate the excess free energy $F_{e x}=W_{1}+V f_{2}$ of the colloids-only sys- tem, with $V f_{2}\left(\eta_{1}, \ldots, \eta_{n}\right)$ the excess free energy of the pairwise Yukawa fluid with Hamiltonian $W_{2}$. This is done here using the Gibbs-Bogolyubov (GB) inequality, with a reference hard-sphere mixture with variational hard-core diameters $d_{i}$ for species $i$, such that $f_{2}\left(\eta_{1}, \ldots, \eta_{n}\right)$ is the minimum of

$$
f_{H S}(\{x\})+2 \pi \sum_{i, j}^{n} \rho_{i} \rho_{j} \int_{d_{i j}}^{\infty} d r r^{2} g_{i j}(r ;\{x\}) v_{i j}(r),
$$

where $x_{i}=(\pi / 6) \rho_{i} d_{i}^{3}$ is the variational packing fraction of species $i, f_{H S}$ is the hard-sphere mixture free-energy density as given in Ref. [17], $d_{i j}=\left(d_{i}+d_{j}\right) / 2$, and $g_{i j}(r)$ are the reference hard-sphere radial distribution functions treated within the Percus-Yevick approximation. Note that expression (3) is explicitly known analytically [17] and its minimization with respect to the variational diameters was performed numerically.

The final step in the calculation of $\mu_{i, e x}$ stems from the thermodynamic identity $\mu_{i, e x}=\partial F_{e x} / \partial N_{i}$. This can be rewritten as $\mu_{i, e x}=\partial\left(w_{1}+f_{2}\right) / \partial \rho_{i}$ and can be calculated numerically using Eqs. (2) and (3). Hence we can solve the hydrostatic equilibrium conditions (1) numerically on a $z$ grid. Moreover, we not only used the EM described by $W=W_{1}+W_{2}$ as an intermediate step in the calculation of $\mu_{i, e x}$, but we also perform direct MC simulations of the EM in Earth's gravitational field. In such MC calculations we actually test the accuracy of the variational GB procedure. The MC simulations using the EM are at least 10 times faster than those of the equivalent PM for the values considered in this paper, because of the absence of explicit ions. By comparing colloidal profiles of the PM and EM one tests in fact the accuracy of our expressions for $W$. One should be aware, however, of a potential problem in MC simulations of the inhomogeneous EM, since both $W_{1}$ and the screening parameter $\bar{\kappa}$ of the pair potentials of $W_{2}$ depend on the colloidal densities, for which we take local densities in slices of a thickness $H / N_{H}$ with typically $N_{H}=200$ in the calculations presented below. Thus MC trial moves in which colloids are displaced from one slice to another involve acceptance probabilities that take both the positional change and the density change into account.

In order to reduce the parameter space we consider equalsized colloids with a common diameter $2 a_{i}=2 a \equiv \sigma$ for $i$ $=1, \ldots, n$, a Bjerrum length $\lambda_{B}=0.004 \sigma$, and a sample height $H=100 \sigma$. The computational details of our PM simulations are given in Ref. [11]. Our EM and YM simulations are based on systems with about 1000-2000 colloids, equilibrated for about $10^{6} \mathrm{MC}$ cycles while averages are taken over $5 \times 10^{5}$ cycles (where 1 cycle contains on average 1 trial move for each particle).

In Fig. 1 we consider a one-component case $n=1$. We find excellent agreement between PM simulations, EM simulations, and EM calculations based on the hydrostatic equilibrium condition (1), except perhaps close to the walls at $z$ $=0$ and $z=H$, where the local density approximation fails to describe the structure. Interestingly $\eta_{1}(z)$ is different (dotted curves) for the YM; i.e., the EM with its volume terms is qualitatively different from the YM. In fact, one checks that 


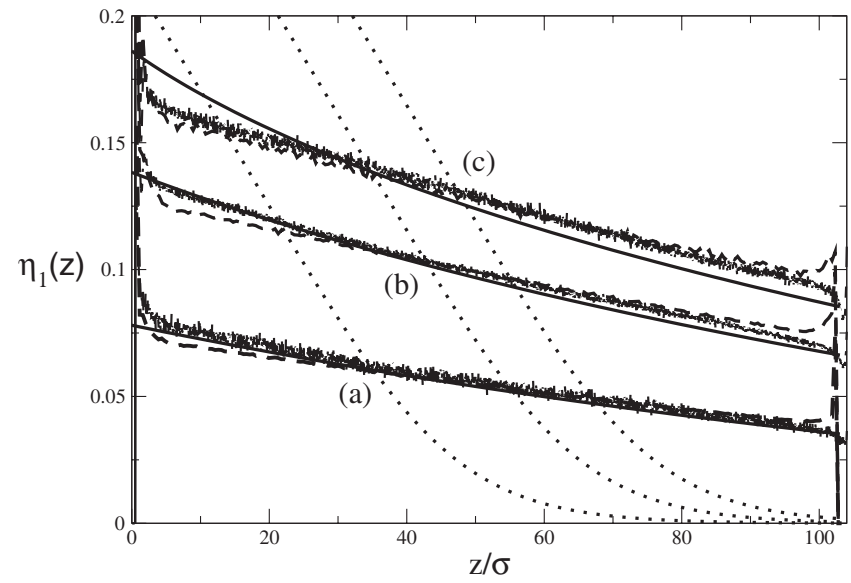

FIG. 1. Packing fraction profiles $\eta_{1}(z)$ for de-ionized monodisperse suspensions of colloidal spheres with diameter $\sigma$, gravitational length $L_{1}=10 \sigma$, charge $Z_{1}=10$, and sample height $H=100 \sigma$, for average packing fractions $\bar{\eta}_{1}=0.055$ (a), 0.099 (b), and 0.128 (c). The PM simulations (noisy curves, taken from [11]), the EM simulations (dashed curves), and the EM calculations based on hydrostatic equilibrium (solid curves) are all in good agreement, while the YM calculations (dotted curves) clearly differ. This indicates the importance of the volume term in the EM.

the volume terms contain a net repulsive contribution here, as the actual profiles extend to higher altitudes than the pairwise ones. This can be traced back to the counterion idealgas contribution in Eq. (2), which is entirely omitted in the YM.

In Fig. 2 we also show quantitative agreement between direct PM simulations, EM simulations, and EM calculations based on hydrostatic equilibrium, but now for density pro-

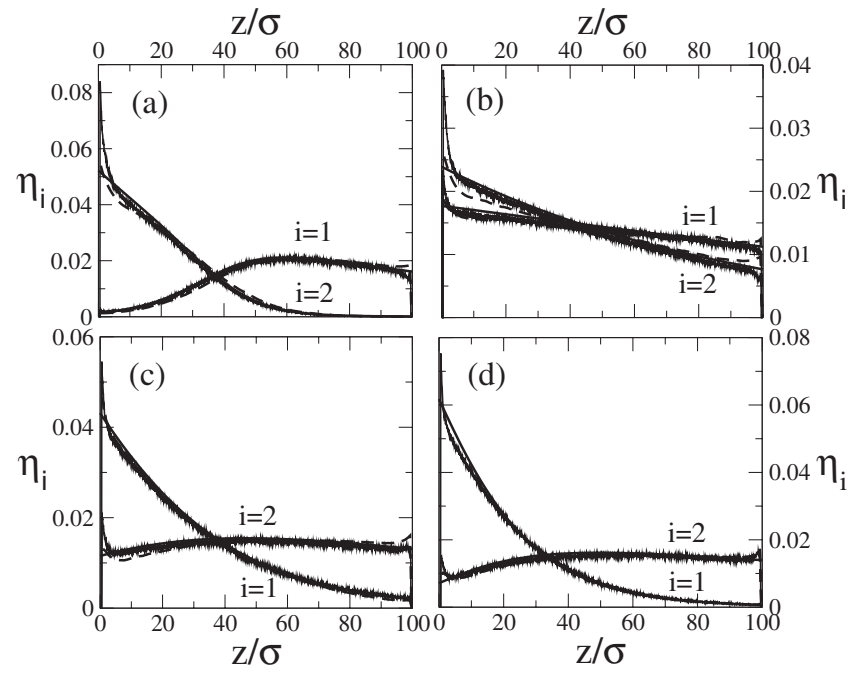

FIG. 2. Sedimentation profiles for a de-ionized binary mixture of equal-sized colloids with diameter $\sigma$, gravitational lengths $L_{1}$ $=10 \sigma$ and $L_{2}=5 \sigma$, average packing fractions $\bar{\eta}_{1}=\bar{\eta}_{2}=0.0144$, and colloidal charges $Z_{1}=10$ and (a) $Z_{2}=10$, (b) $Z_{2}=20$, (c) $Z_{2}=30$, and (d) $Z_{2}=40$. There is quantitative agreement, in all cases, between the PM simulations (noisy curves), EM simulations (dashed curves), and EM calculations based on hydrostatic equilibrium (solid curves).

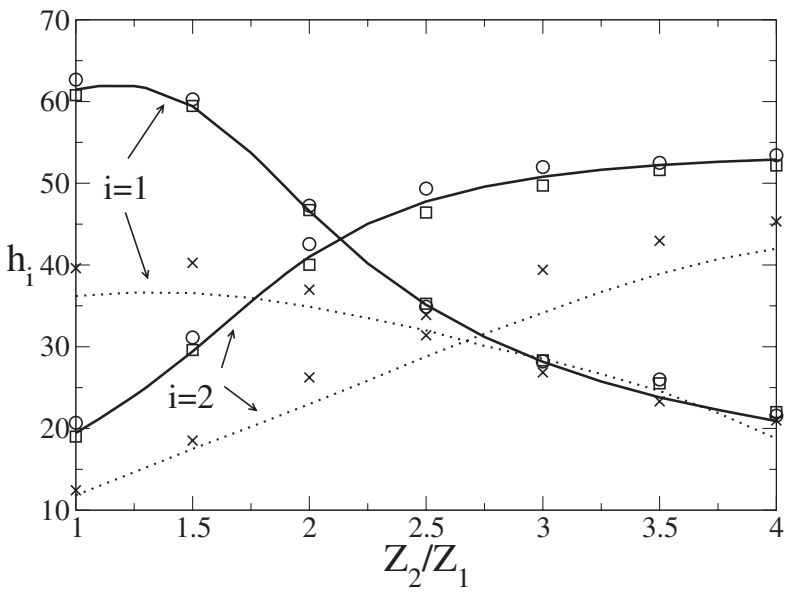

FIG. 3. Mean height $h_{i}$ (see text) as a function of the colloidal charge ratio $Z_{2} / Z_{1}$ for binary mixtures with the parameters given in Fig. 2, showing mutual quantitative agreement between PM simulations (squares), EM simulations (circles), and EM calculations (solid curves). The mean height of the YM from simulation (crosses) and hydrostatic equilibrium calculations (dashed curves) cannot account for the PM results.

files of a two-component system, $n=2$. Figures 2(a), 2(c), and 2(d) show a clear layering effect, in (a) the lighter ones are on top of the heavier ones, in (c) and (d) the order of the layers is reversed ("colloidal Brazil nut effect"), and (b) shows the crossover between these two regimes. Ignoring the volume terms in these systems yields profiles (not shown) that deviate from the actual ones in a fashion similar to that in Fig. 1, again pointing to the importance of $w_{1}$. This can be further quantified by considering the mean height $h_{i}$ $=\int_{0}^{H} d z \eta_{i}(z) z /\left(H \bar{\eta}_{i}\right)$ of species $i$, as shown as a function of $Z_{2} / Z_{1}$ in Fig. 3 for the parameters of Fig. 2. Figure 3 shows quantitative agreement between PM simulations (squares), EM simulations (circles), and EM hydrostatic equilibrium

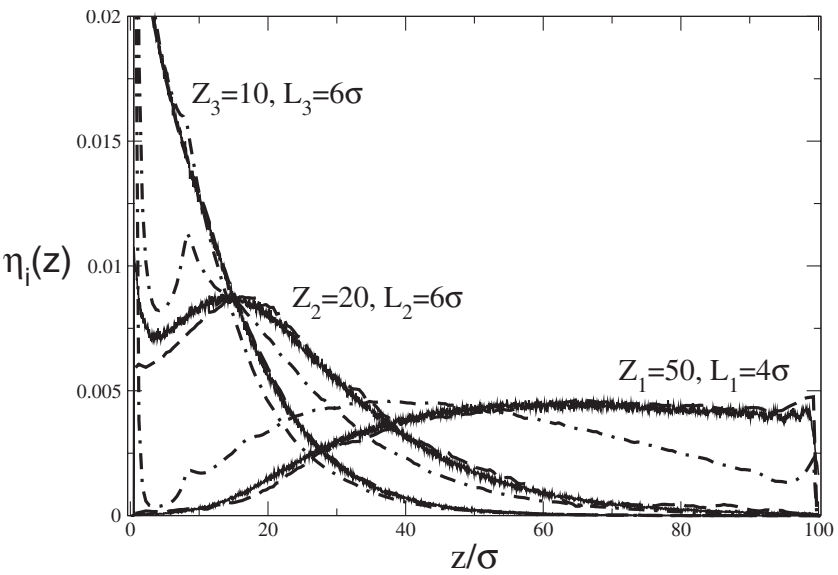

FIG. 4. Sedimentation profiles of a de-ionized equimolar threecomponent colloidal system with average packing fractions $\bar{\eta}_{i}$ $=0.0031$, charges $Z_{i}=50,20,10$, and gravitational lengths $L_{i} / \sigma$ $=4,6,6$ for the three species $i=1,2,3$, respectively, as obtained from PM simulations (noisy curves) and EM simulations (dashed curves) and pairwise Yukawa simulations without volume term (dot-dashed curves). 
calculations (solid curves), while the pairwise YM calculations (dotted lines) and YM simulations (crosses) largely deviate from the other three curves. The mutual deviation between the calculations and the simulations of the YM is to be attributed to the approximate nature of the GB procedure that underlies the calculations. Clearly, Fig. 3 points to the importance of the volume term and to the accuracy with which the PM results are reproduced by the EM, in the present parameter regime at least.

Finally, in order to put the EM to a further test we consider a three-component mixture. The results, shown in Fig. 4 , not only show both layering with increasing mass per charge $\left(\propto Z_{i} L_{i}\right)$ from bottom to top, but also the heavier species 1 floating on top of the lighter species 2 and 3. For these phenomena we find again good agreement between our (previously published [11]) PM simulations (noisy curves) and the EM simulations and calculations (dashed and solid curves, respectively) based on $W=W_{1}+W_{2}$ derived above. Simulations of the pairwise YM (dot-dashed curves) are very accurate for species 3 , but substantially off again for species 1 and 2.

In summary, we have explicitly shown that sedimentationdiffusion equilibrium and effective colloidal interactions in de-ionized suspensions cannot be described by the pairwise Yukawa model for the colloids, not even in the hightemperature limit of interest here. The effective model de- rived here, however, is in quantitative agreement with primitive model simulations. The main difference between the YM and EM is the inclusion of ionic entropy and colloidal selfenergy in the latter-these terms can be ignored at high salt, but turn out to be crucial in de-ionized systems as we show here explicitly. In other words, the EM is an accurate extrapolation of the YM at high salt to the de-ionized regime. The EM that we derived here is based on a linear screening theory and should break down for $Z_{i} \lambda_{B} / a_{i} \gtrsim 5$ because then nonlinear screening effects such as charge renormalization become important [18-20]. The parameters considered in this study are safely in the linear (high-temperature) regime since in all cases $\lambda_{B} / a_{i}<0.01$ and $Z_{i}<100$. So although the presently formulated EM has its restrictions as regards the regime of applicability, it is certainly capable of explaining many-body effects in charged colloids. Moreover, the EM simulations have great computational advantages over those of the PM, since it can reduce the simulation time from months to days.

This work is part of the research program of the "Stichting voor Fundamenteel Onderzoek der Materie" (FOM), which is financially supported by the "Nederlandse Organisatie voor Wetenschappelijk Onderzoek" (NWO). We thank TOP-CW for financial support.
[1] A. E. Bailey et al., Phys. Rev. Lett. 99, 205701 (2007).

[2] R. Piazza, T. Bellini, and V. Degiorgio, Phys. Rev. Lett. 71, 4267 (1993).

[3] M. A. Rutgers, J. H. Dunsmuir, J. Z. Xue, W. B. Russel, and P. M. Chaikin, Phys. Rev. B 53, 5043 (1996).

[4] S. Buzzaccaro, R. Rusconi, and R. Piazza, Phys. Rev. Lett. 99, 098301 (2007).

[5] M. Rasa and A. P. Philipse, Nature (London) 429, 857 (2004).

[6] C. P. Royall et al., J. Phys.: Condens. Matter 17, 2315 (2005).

[7] R. van Roij, J. Phys.: Condens. Matter 15, S3569 (2003); P. M. Biesheuvel, ibid. 16, L499 (2004); A. P. Hynninen et al., Europhys. Lett. 65, 719 (2004).

[8] L. Belloni, J. Chem. Phys. 123, 204705 (2005).

[9] A. Esztermann and H. Löwen, Europhys. Lett. 68, 120 (2004); J. Zwanikken and R. van Roij, ibid. 71, 480 (2005).

[10] A. Torres, A. Cuetos, M. Dijkstra, and R. van Roij, Phys. Rev. E 75, 041405 (2007).

[11] A. Cuetos, A. P. Hynninen, J. Zwanikken, R. van Roij, and M. Dijkstra, Phys. Rev. E 73, 061402 (2006).

[12] M. Dijkstra et al., J. Phys.: Condens. Matter 18, 825 (2006).
[13] C. Russ, H. H. von Grunberg, M. Dijkstra, and R. van Roij, Phys. Rev. E 66, 011402 (2002); C. Russ et al., Europhys. Lett. 69, 468 (2005); A. P. Hynninen et al., J. Phys.: Condens. Matter 15, S3549 (2003); A. P. Hynninen, M. Dijkstra, and R. van Roij, Phys. Rev. E 69, 061407 (2004).

[14] B. Beresford-Smith et al., J. Colloid Interface Sci. 105, 216 (1985); R. van Roij and J.-P. Hansen, Phys. Rev. Lett. 79, 3082 (1997); P. B. Warren, J. Chem. Phys. 112, 4683 (2000); B. Lu and A. R. Denton, Phys. Rev. E 75, 061403 (2007).

[15] T. Biben et al., J. Chem. Phys. 98, 7330 (1993); M. Schmidt et al., J. Phys.: Condens. Matter 16, S4185 (2004).

[16] B. Zoetekouw and R. van Roij, Phys. Rev. E 73, 021403 (2006).

[17] T. Boublík et al., Statistical Thermodynamics of Simple Liquids and their Mixtures (Elsevier, New York, 1980).

[18] S. Alexander et al., J. Chem. Phys. 80, 5776 (1984).

[19] E. Trizac, L. Bocquet, and M. Aubouy, Phys. Rev. Lett. 89, 248301 (2002).

[20] B. Zoetekouw and R. van Roij, Phys. Rev. Lett. 97, 258302 (2006). 